\title{
Introduction to the Open Science Practices in Information Systems Research Minitrack
}

\author{
Cathal Doyle \\ Victoria University of \\ Wellington, New Zealand \\ cathal.dovle@vuw.ac.nz
}

\author{
Yi-Te Chiu \\ Victoria University of \\ Wellington, New Zealand \\ vi-te.chiu@vuw.ac.nz
}

\author{
Tadhg Nagle \\ University College \\ Cork, Ireland \\ t.nagle@ucc.ie
}

\author{
Markus Luczak-Roesch \\ Victoria University of Wellington, \\ New Zealand \\ markus.luczak-roesch@vuw.ac.nz
}

\section{Introduction}

In a world that is starting to question critical thought, and the contributions of experts, it's never been more important for scientists to promote research practices that can build more trust in the research they produce. This can be achieved by becoming more transparent and open with their research by adopting open science (OS) practices. OS refers to making scientific knowledge freely available to the public, and is becoming easier with the advent of information technology, the internet, and platforms such as the Open Science Framework (OSF). This has already resulted in scientists in other disciplines adopting these more open practices, which has seen OS growing in prominence in recent years, challenging the traditional scientific process. This provides IS researchers with an opportunity to do the same, opening up the opportunity for their research not just to be more transparent but increase the possibility of it being adopted by practitioners.

OS consists of different concepts such as open access (making published research articles freely available under an open access licence); open data (requiring researchers to make research data publicly available with their submitted papers); open artefact(s) (making artefacts accessible online for free, with an open license to use,modify and reuse); and open peer review (where researchers and reviewers know each others identities) [1]. There are also registered reports (RR), which facilitate a form of peer review that breaks studies into two stages: (i) study pre-registration authors identify a relevant problem and detail their research design, which is submitted for (open) peer review and feedback, (ii) improved research execution - the study is completed with the improved design. An example of such an approach in IS research can be seen in Doyle and Luczak-Roesch [2].

The objective of this minitrack is to give researchers the opportunity to present novel and innovative ways that they are conducting research using the OS concepts outlined above. In doing so we aim to push the boundary of how IS research is conducted and communicated to the community. Indeed, OS should be more transparent, with greater potential to verify, replicate, and freely share the results. Such openness should also provide a platform for creating research that demonstrates proof-of-use [3] as the community strives to become more relevant to practice. We therefore suggest that the community should strongly engage with OS, and in order to do so, we look for both conceptual and empirical papers that either further our understanding of OS in IS research, or studies that practice it.

The paper in this year's minitrack is: "Open Science via HUBzero: Exploring Five Science Gateways Supporting and Growing their Open Science Communities". This paper discusses the open science gateway HUBzero, which is a science gateway framework to support communities through efficient sharing and publication processes. In particular, they focus on five communities (nanoHUB, MyGeoHub, QUBEShub, CUE4CHNG, and HubICL) discussing their features and growth. From this, they offer insights for science gateways to foster open science and open education.

\section{References}

[1] Doyle, C., Luczak-Roesch, M., and Mittal, A. (2019). We Need the Open Artefact: Design Science as a Pathway to Open Science in Information Systems Research. In Proceedings of the International Conference on Design Science Research in Information Systems and Technology 2019, pp. 46-60..

[2] Doyle, C., and Luczak-Roesch, M. (2020). This paper is an artefact: On open science practices in design science research using registered reports. In Proceedings of the 53rd Hawaii International Conference on System Sciences, pp. 5026-5035. 
[3] Nunamaker Jr, J.F., Briggs, R.O., Derrick, D.C. and Schwabe, G., 2015. The last research mile: Achieving both rigor and relevance in information systems research. Journal of Management Information Systems, 32(3), pp.10-47. 